Instituto Internacional de Investigación y Desarrollo Tecnológico Educativo INDTEC, C.A.

DOI: https://doi.org/10.29394/scientific.issn.2542-2987.2017.2.6.12.238-254

OAI-PMH: http://www.indteca.com/ojs/index.php/Revista Scientific/oai

\title{
Cultivo de Bambú como Recurso Natural Renovable para la Reforestación en la Quebrada Echeverría
}

\author{
Autora: Mariela del Carmen López Salazar \\ Universidad Pedagógica Experimental Libertador, UPEL \\ alexmariela9@hotmail.com \\ Barinas, Venezuela
}

\section{Resumen}

La investigación tiene como objetivo, el cultivo del Bambú, la reforestación para la recuperación de la quebrada Echeverría, parroquia Manuel Palacio Fajardo del municipio Barinas, Estado Barinas, dirigido a los habitantes de la comunidad del sector la Caramuca. La metodología es de naturaleza cualitativa en la que se destaca la investigación acción participativa, como vía para acceder a las características particulares del tema de estudio, se enfoca en cinco (5) fases de investigación: puesta en práctica a partir tres planos de experiencias, es decir tres (3) habitantes de la comunidad; a mayoría de ellos conocen las consecuencias que se traen a la práctica, las técnicas de recolección de los información estarán representada por la observación y la entrevista. Análisis de información por categorización, triangulación de fuente e interpretación. En tal sentido se recomienda realizar actividades prácticas de conservación ambiental, para desarrollar actividades del cultivo del bambú donde se desarrolla prácticas educativas acerca de la conservación y reforestación de esta cuenca.

Palabras clave: cultivo del bambú; reforestación; conservación; educación ambiental. 


\title{
Bamboo Cultivation as a Renewable Natural Resource for Reforestation in Echeverría Brokening
}

\begin{abstract}
The research has as objective, the cultivation of Bamboo, the reforestation for the recovery of the Echeverría ravine, Manuel Palacio Fajardo parish of Barinas, Barinas State, aimed at the inhabitants of the community of the Caramuca sector. The methodology is qualitative in which the participatory action research is highlighted, as a way to access the particular characteristics of the study topic, focuses on five (5) research phases: implementation from three levels of experiences, that is to say three (3) inhabitants of the community; most of them know the consequences that are brought to the practice, the techniques of information collection will be represented by observation and interview. Analysis of information by categorization, source triangulation and interpretation. In this sense it is recommended to carry out practical environmental conservation activities to develop bamboo farming activities where educational practices are developed regarding the conservation and reforestation of this basin.
\end{abstract}

Keywords: bamboo farming; reforestation; conservation; environmental education.

Date Received: 07-06-2017

Date Acceptance: 10-08-2017 


\section{Introducción}

En la actualidad, se debe tener en cuenta que la vida humana tiene una estrecha relación con la naturaleza y que su funcionamiento nos afecta totalmente, donde el ambiente es el productor natural que existen; nos permiten vivir del ambiente de su estructura y de su funcionamiento, la cual nos demuestra la profundidad de estas relaciones, debido al conflicto que actualmente se está viviendo. Donde el ser humano siempre ha podido satisfacer sus necesidades biológicas, fisiológicas, socioeconómicas y culturales, que influyen en la vida. No se trata del espacio en el que desarrolla la vida, sino que también comprende seres vivos, las relaciones entre ellos la actualidad se encuentra afectada debido a las grandes demandas del hombre, causando así un desequilibrio en los recursos naturales, es necesario tomar consciencia de esto.

El presente trabajo de investigación tiene por objetivo principal, es la recuperación de la quebrada Echeverri, parroquia Manuel Palacio Fajardo del municipio Barinas Estado Barinas. Se basa en la gran inquietud por la problemática que afecta a la comunidad adyacente a la quebrada de la Caramuca ante la posibilidad de erosión de los suelos circundante de la misma; se desea contribuir a la protección, recuperación y conservación de este ecosistema con actividades de reforestación protectora basada en la siembra de especie (bambú) en la cabecera de la quebrada.

La siguiente publicación se basa en el problema que existe en la comunidad Echeverría, la mencionada quebrada la cual proveer de agua a gran parte de la comunidad, tanto de consumo humano como para el riesgo de sus cultivos. La investigación se impulsó en un paradigma cualitativo, sustentado en un enfoque de campo con un tipo de investigación acción participativa. Uno de los recursos naturales que se encuentra afectado son las cuencas hídricas; dentro de esta misma idea se han tratado temas ecológicos como deforestación- desertización, principal causa destructora de las cuencas. 
Se habla del manejo racional, pero nadie sabe lo que es racional. Se hace una represa que cuesta varios miles de millones de bolívares, y al mismo tiempo se desarrolla una agricultura que causa daño en la cuenca que abastece la represa; de esta manera, a lo mejor la vida de la represa será la mitad del programa, Las ideas expuestas por el citado autor, hacen referencia a la necesidad de que se atienda el problema de la conservación; éste no se puede postergar, ya que se debe actuar en el menor tiempo posible, en virtud a que la demanda de agua aumenta constantemente, mientras que caudales aprovechables varían de manera sensible y la escasez cada día se hace más notoria.

El estado Barinas hay muy poca producción de bambú, y hasta se plantea desconocimiento de la especie por parte de los habitantes de muchas zonas rurales que se sitúan en áreas aledañas a fuentes o cabeceras de ríos y quebradas. Por esta razón tomo como iniciativa del trabajo de investigación la producción de bambú para reforestar un sector en las orillas de la quebrada la Caramuca, ubicada en la Parroquia Manuel Palacio Fajardo del Municipio Barinas; donde se aprecia la falta de forestación causando que la quebrada pierda su cauce y que las erosiones de los suelos circundantes pierdan la fertilidad para la producción agropecuaria. De allí surge la inquietud de realizar un proyecto, que además de beneficiar a la comunidad en general será de gran ayuda para el ambiente.

Basados en la situación antes planteada y por la inquietud de desarrollar un proyecto de producción agroforestal del bambú para prevenir la sequía de una fuente natural de agua.

\section{Teoría y Concepto}

\subsection{La Reforestación}

El concepto de reforestación es la siembra de árbol, que se realiza con la finalidad de conservar un terreno o espacio, donde su importancia es 
asegurar la protección del bosque. Se puede decir, que por los avances tecnológicos, los vehículos, las construcciones, la industrialización, que está causando daño a nuestro planeta donde no toma en cuenta medidas de precaución para evitar la deforestación.

Se puede decir que la reforestación sirve para la supervivencia del hombre y de los seres vivos, teniendo en cuenta la gran cantidad de talas e incendios, que está destruyendo la selva, el oxígeno se va perdiendo poco a poco.

De igual forma, Ruiz (2002), en el Manual de Reforestación para América Tropical, expresa:

"El corte indiscriminado y a gran escala reduce los recursos genéticos y la diversidad de especies y puede llevar a la extinción de la flora y fauna local, en áreas donde no se han realizado inventarios de los recursos del bosque y de la vida silvestres, estas pérdidas pueden pasar desapercibidas; otro gran problema lo constituyen las alteraciones a los suelos, ya que se remueven grandes cantidades de materia orgánica directamente en forma de madera o se alteran las mismas mediante la agricultura tipo corte y quema" (pág. 5).

Con este argumento, nos lleva a la inquietud por el gran deterioro del hábitat de diversos aspectos conlleva la descomposición orgánica que disminuye enormemente, causando la disgregación de las partículas de tierra, la pérdida de la capacidad para retener la humedad y fertilidad del suelo; el terreno así expuesto se desintegra fácilmente por el impacto de la lluvia, rodándose y siendo llevado por las escorrentías a las quebradas y ríos.

\subsection{La Deforestación}

La población mundial está creciendo en forma descomunal que satisface sus necesidades, obliga mayor explotación de recursos. Asimismo, se va destruyendo el clima, la principal causa de deforestación es la explotación de madera, para convertirlos en ciudades, industrias y carreteras 
o vías, donde no es tomado en cuenta el daño que se le hace al ambiente y las consecuencias que nos trae más adelante a los futuros herederos. Nuestros ríos se encuentras afectado, por la tala del bosque y en las cuencas, debido a los crecimientos repentinos que afecta a la fauna.

Lo anteriormente citado infiere en la urgente necesidad de diseñar y desarrollar programas, con el fin de proteger, conservar y hacer buen uso de los recursos hídricos, sin alterar ni destruir la vegetación cercana a las cuencas.

\subsection{Educación Ambiental}

Cada día el proceso de formación o educación ambiental, logra informar sobre el cuidado del ambiente. El proceso de formación permite reflexionar la importancia del ambiente que nos rodea, en el que promueve a la naturaleza el progreso de servicios y nuevas cualidades que ayuden al uso racional de los recursos naturales y a la solución de los problemas ambientales.

La conservación del ambiente consiste en el uso racional de los recursos que nos brinda la naturaleza, para lograr un desarrollo sostenible que garantice la vida de las generaciones futuras. Hoy en día nuestro planeta está sin agua, sin tierras fértiles para cultivar, sin un aire puro, es por ello es importante que conservemos el ambiente para nuestros hijos.

Se puede decir que para puede ayudar a contribuir a la educación ambiental es:

El Estado con leyes que tenga que ver con la educación ambiental y protección del ambiente; asignar presupuestos adecuados para programas educativos.

La comunidad puede desarrollar y participar activamente en los programas. 


\subsection{Participación Comunitaria}

La participación de las comunidades en conservación es muy importante fundamentalmente en la conservación de las cuencas hídricas; ya que es trascendental educar y sensibilizar a la población sobre la importancia de este vital líquido para que no se contamine ni se dilapide, se deben fijar estrategias que conduzcan a reducir la aparición de incendios de vegetación, lograr un cambio de actitud favorable a la conservación de los recursos, al mantenimiento o recuperación del equilibrio ecológico de los sistemas naturales.

A tal fin, el sector educativo en todas sus modalidades, debe convertirse en un foco de conciencia ecológica, que interese a todos los estratos sociales y a todas las actividades humanas; logrando de esta manera, un cambio de actitudes con respecto al entorno donde se desenvuelven los individuos y las comunidades y adquieran los conocimientos, los valores y la experiencia que les permitirá actuar individualmente y colectivamente, en la resolución de problemas actuales y futuros del medio ambiente, logrando impulsar un proceso de desarrollo integral que no destruya la base de un recurso natural y que tenga como meta el mejoramiento de la calidad de vida.

\subsection{Cuencas Hídricas}

La cuencas hidrográficas, áreas de escurrimiento superficial que drenan hacia un cauce común que vierten sus aguas a través de una sección específica o punto de concentración constituyen unidades físicas, independientes en la mayoría de los casos donde se desarrollan tanto los eventos permanentes de la naturaleza, fenómenos climáticos hidrológicos, biológicos, geomorfológicos como las actividades usuales del hombre, es decir, sociales, económicas y culturales, a partir de la interrelación entre las condiciones físico naturales de una cuenca y de la intervención antrópica en la misma, se genera un medioambiente característico de cada una, en el cual, 
para la supervivencia humana es necesario un aprovechamiento y uso de los mismos de manera armoniosa, equilibrada bajo la consideración de realizar un verdadero desarrollo sustentable.

Por ello, la conservación de las cuencas representa una necesidad impostergable, debido a la importancia esencial que tienen los ríos quebradas para la humanidad, pues desde tiempos remotos ha servido como medio de transporte, para riego y principalmente satisfacer las condiciones primarias del hombre, en cuanto a producción y alimentación; ya que cada vez es mayor la dependencia de los seres humanos, porque ésta permite realizar diversas actividades en los cuales se aprovecha el recurso, se usa, transforma, consume y disfruta. Las cuencas reciben agua en forma de precipitaciones como parte del agua (ciclo hidrológico), algunas precipitaciones regresan a la atmósfera una vez que han sido captadas por la vegetación y se han evaporado en la superficie de las hojas y ramas, la mayor parte de pierde por la evaporación que tiene lugar en el suelo y por la transpiración de las plantas.

En los climas áridos y semiáridos es habitual que todas las precipitaciones se consuman de esta forma; la escorrentía sólo ocurre en ocasiones, después de fuertes tormentas. La escorrentía depende además de la tasa de evaporación, de la pendiente del terreno, de la naturaleza de las rocas y de la presencia 0 ausencia de manto vegetal. Cuando las precipitaciones superan a la pérdida debido a la evaporación y transpiración, el excedente de agua sigue su curso en el sistema de drenaje y corre sobre la superficie del terreno; sin embargo, su avance no es uniforme, es posible que las aguas se acumulen en lagos, suelos, o como parte de las aguas subterráneas durante largos períodos antes de fluir finalmente como escorrentía hasta alcanzar el canal de la cuenca.

Las aguas que se infiltran y alcanzan la zona de aguas subterráneas permanentemente saturadas (agua freática), por debajo del nivel freático actúan como caudal basal de los ríos de la cuenca; las aguas de la zona 
parcialmente saturada influyen por acción de la gravedad, por el suelo y por las áreas situadas por encima del nivel freático. Las aguas que no logran filtrarse en el suelo discurren por la superficie. La proporción de escorrentía que sigue estos diferentes caminos depende de diversos aspectos; algunos de los cuales son propiedades permanentes de las cuencas (estructura y relieve), mientras que otros factores pueden variar con el tiempo o como consecuencia de las actividades del hombre (clima, suelo y vegetación), y en función de la meteorología reciente de la cuenca.

El drenaje del subsuelo facilitado por el agua vadosa y por las aguas subterráneas, se produce más lentamente que el drenaje ocasionado por las aguas superficiales, un aspecto significativo a la hora de considerar el caudal basal del sistema fluvial, junto con los aportes de las precipitaciones. Cuando la escorrentía se concentra, la superficie terrestre se erosiona, creando un canal, lo cual forma una red que recoge las aguas de toda la cuenca y las vierte en un único río que se halla en la red, pero la estructura geológica subyacente suele ser el factor más relevante, los patrones hidrográficos se encuentran íntimamente relacionados con la geología, lo que permite identificar cualquier falla.

En este sentido, esta parte constituye el lugar donde afluyen las diferentes aguas y desembocan cauces principales de ríos, lagos.

\subsection{Desarrollo Sustentable}

Obedece a las necesidades de crecimiento social, económico de la sociedad, con el fin de desarrollar estrategias que toma aspectos cualitativos, donde ha sido discutida por diferentes países.

Por lo tanto, para conseguir un desarrollo sustentable es necesario un cambio social con diversas virtudes de crear, desarrollar, implementar en el campo de estudio. 
Así mismo, cuando se aborda el problema de la sustentabilidad es importante señalar que los recursos son sustituibles unos por otros, la madera y la piedra fueron sustituidos por concreto y cabilla, de manera que el agotar un recurso, sobre todo cuando es no renovable, no es necesariamente incorrecto o injusto con las generaciones futuras, si como resultado del uso de esos recursos el ambiente es mejorado.

En este orden de ideas, se puede decir que en la medida que se aproveche utilice racionalmente los recursos, se estará contribuyendo con el desarrollo sustentable, al mismo tiempo que se obtendrá beneficios y mejorará la calidad de vida. Por ello, el desarrollo sostenible constituye un proceso de cambio social en el cual la explotación de los recursos, el sentido de las inversiones, la orientación del desarrollo tecnológico, se realizan en forma armónica, desarrollar legal, actual y futuro para integrar las necesidades y deseos humano.

Lo anteriormente expuesto constituye una de las formas más convenientes que generan sustentabilidad, ya que se hace imprescindible invertir en la conservación de los recursos naturales; de esta forma se estará contribuyendo con las generaciones futuras, las cuales exigirán que el desarrollo sea compatible con el mantenimiento de los procesos ecológicos y fortalecimiento de la identidad en las comunidades, logrando un equilibrio demográfico en el cual se dé un desarrollo económicamente eficiente y equitativo dentro y entre generaciones.

\section{Bases Legales:}

A continuación, se presentan basamentos legales, sobre los cuales se realiza la presente investigación, que involucran al colectivo de la sociedad para el logro de sus objetivos. A través de los cuerpos normativos y dispositivos más relevantes tales como: 
La Constitución de la República Bolivariana de Venezuela (1999), en el capítulo VI referente a los derechos culturales y educativos, en el artículo 107 establece "La educación ambiental es obligatoria".

En este mismo orden de ideas, es imperativo que todas las personas se instruyan acerca de la conservación ambiental y se cree conciencia ecológica, desde los primeros años de estudio, y los ciudadanos que no cursen educación formal, están en la obligación de cultivarse en cuanto a lo concerniente a la protección del ambiente.

En este sentido, la Constitución Nacional plantea la necesidad de que cada ciudadano proteja y conserve el ambiente; de esta manera obtener beneficios a corto y largo plazo. Además, el estado tendrá la obligación de resguardar la biodiversidad biológica, así mismo garantizarle a la población un medio libre de contaminación.

\subsection{Ley del Plan de la Patria (2013)}

Las Políticas y programas del Sector Ambiente Conservación y ordenación territorial:

El plan de la patria busca implementar una política que se encargue de resguardar nuestra naturaleza para provechar el uso racional de los recursos naturales, buscando tener conciencia ciudadana, por eso es importante realizar proyecto que sustentable ya esto no compromete el medio ambiente sino satisface las necesidades presentes en una comunidad, combinando armonio aspectos sociales, ecológicos y económicos.

\subsection{Ley Orgánica del Ambiente (2012)}

En su artículo Establece los derechos de conservar, para que el recurso natural se mantenga un ambiente sano, seguro, para así prevenir problemas ambientales que están actualmente en la comunidad. Expresa sobre los usos de los espacios y condiciones de especies para su conservación, que existe 
normas que oriente a los procesos para la mejora del ambiente no para su destrucción, el estado garantiza la incorporación de dimensiones ambientales como planes urbanísticos, industriales, comerciales entre otros.

La educación ambiental es unos de los lineamientos más importantes que se encuentra en esta ley, ya que es de suma importancia porque en ella incorpora las unidades educativas y la comunidad en general para implementar conocimiento, En que cada uno protege y mantiene el ambiente.

\section{Metodología}

La metodología planteada en la investigación considera el uso de técnicas y procesos estadísticos para diagnosticar e inferir ciertos comportamientos observables en la población objeto de estudio y que servirán para buscar una solución efectiva a la problemática planteada.

El marco metodológico de una investigación que comprende las vías a seguir desde que se inicia el estudio hasta su culminación, el conjunto de procedimientos lógicos, técnicos y operacionales implícitos en todo proceso de investigación, La presente investigación está orientada bajo la modalidad cualitativa, donde la misma se sustenta en la investigación de naturaleza profunda de la realidad, su estructura dinámica como lo expresa, Martínez (2008a), "el enfoque cualitativo es sensible a la complejidad de la vida humana”. (pág. 104). En consecuencia, el enfoque persigue penetrar de manera rigorosa y sistemática en los fenómenos de la vida cotidiana, explorarlo, analizarlo para mostrar su complejidad.

Es importante destacar que la investigación cualitativa estudia los fenómenos desde su propia realidad, como un todo integrado desde su naturaleza, sin considerar las partes separadas una de las otras de forma sistemática y dialéctica. 


\subsection{Diseño y Tipo de Investigación}

El diseño de investigación se basará en un enfoque de campo y se conducirá en el propio sitio donde se encuentra el universo estudiado. En este caso, Fidias Arias (2012), define: "la investigación de campo como aquella que consiste en la recolección de datos directamente de los sujetos investigados o la realidad donde ocurre los hechos sin manipular o controlar la variable". (pág. 31).

La investigación de campo surge como una estrategia para cumplir con métodos que permiten recoger los datos en forma directa de la realidad donde se presenta.

En es una investigación acción participativa donde se cumplirá con las siguientes etapas generales: diagnóstico, planteamiento y fundamentación teórica de la propuesta; procedimiento metodológico, actividades y recursos necesarios para su ejecución; análisis y conclusiones sobre la investigación.

En este sentido, como el estudio se ubica en acción participación, el cual es definido por Martínez (2008b), como "aquel tipo de investigación que trata de una propuesta de acción para resolver un problema práctico o satisfacer una necesidad". (pág. 242). Trata de buscar la solución a la necesidad del entorno social, el caso puntual de esta investigación está enmarcada en la siembra de la especie vegetal Bambú, como recurso natural renovable para reforestar de la quebrada de la Caramuca, a fin de prevenir la sequía total de la mencionada quebrada, de esta manera contribuir al cuidado del ambiente y mejora la calidad de vida de las comunidades aledañas.

Para desarrollar la investigación se seleccionaron informantes clasificados, considerados como aquellas personas que se relacionan de manera directa con el entorno, en este caso son tres (3). Habitantes cercanos de la quebrada, lo cual tienen conocimiento de la realidad latente.

Entre las técnicas empleadas para tal fin, se utilizó la observación y se emplea la entrevista, que Martínez (2008c), "técnica aplicada, clásica, primaria 
y más usada por los investigadores cualitativos para adquirir información". (pág. 308).

La observación permite la descripción de algunas actividades realizadas por los habitantes de la comunidad y se toma notas de campo relacionada con los hechos observados durante las visitas, además se toma fotografía donde se evidencia la situación presente, esto con la finalidad de apreciar la situación presente; la observación, constituye una herramienta indispensable para la indagación.

De igual manera se emplea la entrevista, con el fin de obtener más información directamente de los habitantes del área a estudiar, porque precisamente son los habitantes de la comunidad quienes mejor conoce la problemática en la están inmersos, aportando de esta manera información relevante para la aproximación de objeto de estudio.

Al respecto Martínez (2008d), define "la entrevista como la técnica clásica primaria y más usada por los investigadores cualitativos para adquirir información" (pág. 89). Además, el autor nos revela, que dicha técnica es el paso más común para los estudios cualitativos, por lo que ofrecen más veracidad, certeza y seguridad en una investigación cualitativa.

\section{Conclusión}

La mayoría de los habitantes del sector, están informados que la quebrada de la Caramuca está en un proceso de erosión, además están conscientes de que esto perjudica a toda la población; por cuanto el interés principal al ejecutar esta investigación es una forma de aporta una solución que persigue aliviar el daño causado por la desforestación aplicada en la zona. La tala y la quema perjudican enormemente a la naciente ya que en los últimos años se han venido observando un deterioro en la cuenca lo cual to ha ocasionado la disminución severa en el caudal de la quebrada. 
Esto surge a raíz de la eliminación progresiva de los bosques naturales, lo cual trae como efecto la erosión de la naciente de agua y el incremento en el proceso de erosión de las tierras. En función del cultivo de algunas planta (bambú) como recurso natural renovable donde los habitantes conoce varias planta y sabe algunas que aporta un gran beneficio para la naciente de agua de diferentes característica botánicas y biológicas que dan beneficio al medio ambiente, aportado control de la erosión, es particularmente adecuado para plantaciones forestales por un corto periodo vegetativo, también se propone como un ecosistema donde se podrá convivir abundante flora y fauna, diversidad de insectos, aves y hasta anfibios.

De acuerdo con lo ya planteado se destaca la necesidad de realizar esta investigación ya que será beneficiara a varias comunidades de la Caramuca, los mangos, brisa y Echevarría, así brindarle una mejor calidad de vida, que permitan lo antes posibles desacelerar el proceso del cambio climático, convirtiéndose la producción agroforestal en algunas de las alternativas que se necesitan para realmente impactar en la reducción del daño ambiental que en este momento genera dicha comunidad.

\section{Referencias}

Constitución de la República Bolivariana de Venezuela (1999). Gaceta Oficial Nro. 36860, 30 de diciembre 1999. Caracas, Venezuela. [Documento en línea]. Recuperado de:

http://www.ucv.ve/fileadmin/user upload/auditoria interna/Archivos/Ma terial de Descarga/Constitucion de la Republica Bolivariana de Ve nezuela - 36.860.pdf

Fidias, A, G (2012). El proyecto de investigación. Introducción a la Metodología Científica. 6ta Edición, Editorial Episteme, Caracas.

Ley Orgánica del Ambiente (2006). Gaceta Oficial Nro. 5833 Extraordinaria. 22 de diciembre. Caracas, Venezuela. Recuperado de: 
https://es.scribd.com/doc/47588555/ley-organica-del-ambiente Ley del Plan de la Patria (2013). Segundo Plan Socialista de Desarrollo Económico y Social de la Nación 2013-2019. Publicado en Gaceta Oficial de la República Bolivariana de Venezuela. Nro. 6.118 Extraordinario, de 2013. Recuperado de:

http://www.mppp.gob.ve/wp-

content/uploads/2013/09/ley plan patria.pdf

Martínez M. M. (2008a,b,c,d). Ciencia y arte en la Metodología Cualitativa. México: Trillas.

Ruiz, (2002). Manual de Reforestación para América Tropical. Servicio Forestal del Departamento de Agricultura de los Estados Unidos. Puerto Rico. Recuperado de: http://www.bio-nica.info/biblioteca/Ruiz2002.pdf 
Mariela del Carmen López Salazar

e-mail: alexmariela 9@hotmail.com

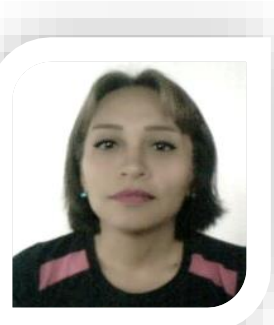

Nacida en Barinas, Estado Barinas, Venezuela. Cursa estudios de maestría en educación ambiente y desarrollo en la Universidad Pedagógica Experimental Libertador, núcleo Barinas. Profesora en Educación Integral en la UPEL Barinas. T.S.U. en Educación Mención Integral.

Actualmente me desempeño como Profesora en Unidad Educativa Nacional de Talentos Deportivos, Barinas del Estado Barinas. Con participación en el Proyecto Pedagógico de Aula, Taller Orientaciones Técnicas Metodológicas para la producción de soluciones Educativas Computarizadas, Curso en el uso Educativo de las tecnologías de la información y la comunicación, Uso de la Tecnología y La Informática (TIC), Introducción a la Computación e Informática, Inducción Docente Modalidad a Distancia, Curso de Asistente Jurídico, Juegos, Dinámicas y Canciones, II Jornada de Educación Inicial, Curso de Linux Versión KNOPPIX 3.7, Producciones Pedagógicas, I Feria Edumatica, II Feria Edumatica, Jornada: Practicas Practicantes y Producción Pedagógica. Desempeño laboral Docente Interino de aula en U.E. Fe y Alegría "La Inmaculada" Barinas, Instructora de Cátedra Bolivariana y Cooperativismo INCES, Barinas.

El contenido de este manuscrito se difunde bajo una Licencia de Creative Commons ReconocimientoNoComercial-Compartirlgual 4.0 Internacional 